\title{
Comparison of image quality of an abdominal acquisition mode of angiography systems from four major manufacturers
}

\author{
Lucie Sukupova*1, Jan Rydlo², Ondrej Hlavacek², Daniel Vedlich², Jan H. Peregrin ${ }^{2}$ \\ ${ }^{1}$ Director Department, Institute for Clinical and Experimental Medicine, Prague, Czech Republic \\ ${ }^{2}$ Department of Radiodiagnostic and Interventional Radiology, Institute for Clinical and Experimental Medicine, Prague, Czech \\ Republic
}

Received: April 12, 2018

DOI: $10.5430 /$ ijdi.v5n2p6
Accepted: July 3, 2018

Online Published: August 13, 2018

\begin{abstract}
Objective: The aim of this study was to compare image quality of different abdominal acquisition modes under conditions simulating obese patients whose images suffer more from noise and scatter radiation. Images were acquired in clinically used acquisition modes on the static and dynamic phantom for four angiography systems.

Methods: A LEGO cart with $34 \mathrm{~cm}$ of PMMA and Pro-RTG Fluo18 phantom were used to simulate obese patients. The low-contrast resolution was assessed subjectively by two readers and objectively using signal-difference-to-noise ratio (SDNR) and using SDNR to air kerma rate. The line-pair resolution was assessed using the transmitted contrast value for line-pair groups. Results: Systems use different exposure parameters and dose but they differ in postprocessing too. Qualitative and quantitative assessments of noise produced similar results, images produced by systems A and C were noisier than by systems B and D. Highest SDNR was provided by System B, whilst System A produced the lowest values, which were almost the same for objects with different contrast. The image quality was affected mainly by frame lengths and postprocessing, but also by the dose. The images of the static phantom were better compared to the images of the dynamic phantom, which was an expected result.

Conclusions: It was possible to identify image quality differences and to characterize features of postprocessing from measurements on standardized objects. A potential for optimization on some systems was identified, although further work, including assessment of clinical images, would be needed as part of the optimization process.
\end{abstract}

Key Words: Angiography, Dynamic phantom, Image quality, Obese patient

\section{INTRODUCTION}

In recent decades, the number of obese patients undergoing interventional procedures has increased. ${ }^{[1]}$ The image quality of obese patients is quite different from the image quality of low or normal weight patients. These images suffer from much higher noise and scatter radiation, which affect visibility of low-contrast objects. ${ }^{[2]}$ The images of obese patients may also be affected by the motion unsharpness due to the demand for more X-ray photons, which are produced by longer frames (pulses).

The exposure parameters and consequently the dose (rate) of current angiography systems are controlled by the automatic dose rate control (ADRC). ${ }^{[3]}$ The role of the ADRC is to modify the exposure parameters in such a way that the

\footnotetext{
*Correspondence: Lucie Sukupova; Email: lucie.sukupova@gmail.com; Address: Institute for Clinical and Experimental Medicine, Prague, Czech Republic.
} 
energy of X-ray photons absorbed in the flat panel detector corresponds to the preset value.

Parameters that are modified by the ADRC according to attenuation of the examined object are the tube current, frame length, voltage, spectral filtration and focus size, but not all systems permit all these five parameters to be varied simultaneously. ${ }^{[4]}$ A combination of the parameters depends on the imaging mode (cardiology or radiology, aorta or limbs etc.), the range of allowed values (predefined parameter space) and the system/manufacturer. Some systems use signal-to-noise ratio and some use contrast-to-noise ratio. The functioning of the ADRC for angiography systems is described in the literature. ${ }^{[3,5-7]}$

The frame length is chosen in such a way that the motion unsharpness is minimized (the frame length for the imaging of limb arteries would be too long for the imaging of coronary arteries). Higher voltage and higher spectral filtration can decrease the image contrast, but postprocessing may compensate for this, so it might not be apparent to the image observer. Different mathematical operations are performed in the images, usually temporal and spatial filtration, but also temporal averaging. ${ }^{[8,9]}$ Each manufacturer uses different postprocessing software, e.g. Advanced Image Processing with Super noise reduction filter (Toshiba), ClarityIQ technology (Philips) or CLEAR+CARE technology (Siemens). ${ }^{[10-12]}$

Thus the attenuation of the patient, preset detector dose value, performance of the X-ray tube and postprocessing are the main factors that influence the patient's dose and image quality. ${ }^{[3,6,7]}$

Assessment of image quality of patient images is usually subjective, because it relies on human perception, albeit of highly trained individuals. Many of physical image quality tests are also subjective, and prone to inter- and intra-observer variability. For an objective comparison, a quantification of image quality is necessary. Basic quantitative measurements using standard image quality phantoms that include low-contrast and high-contrast objects may be obtained using software-based measurements and calculations. ${ }^{[13]}$

The aim of this study was to compare the image quality as regards noise and low- and high-contrast resolution of the selected acquisition mode, which is used clinically for abdominal procedures, on different angiography systems under conditions simulating obese patients. For these patients, the image quality is expected to be affected by large attenuation, causing higher scatter, and the system may reach the limiting values of exposure parameters, resulting in poorer image quality.
Images were acquired in clinically used acquisition modes including automatic detector corrections and image postprocessing. There are pros and cons to this approach, the main advantage being that it gives some insight into the influence of clinical image processing. This is increasingly important if measurements are to be relevant to the clinical setting. Raw data mode is preferred for some types of objective metrics when non-anthropomorphic phantoms are used, but for tests described in this paper, requirements such as images being linear shift invariant etc. were not required.

\section{Methods}

The abdominal area of the obese patients might have a sagittal abdominal diameter of $35-40 \mathrm{~cm}$ (in the posteroanterior [PA] direction). ${ }^{[14-16]}$ For the simulation of the patient's attenuation of the abdominal area, $34 \mathrm{~cm}$ of the polymethyl methacrylate (PMMA) was used, which corresponds to the $38 \mathrm{~cm}$ of the soft tissue. ${ }^{[17]}$

The exposure conditions of obese patients were simulated not only by a static phantom but also by a dynamic phantom, to show differences when the structures are not static. The dynamic phantom (the moving object) was simulated by a LEGO cart (see Figure 1) with the low- and high-contrast object phantom Pro-RTG Fluo18 (Pro-Project, Poland, Figure 2). The cart joint to the motorized engine moved with a constant velocity of $0.8 \mathrm{~cm} / \mathrm{s}$. This velocity was sufficient to simulate the motion of abdominal organs when a patient holds his breath. ${ }^{[18]}$

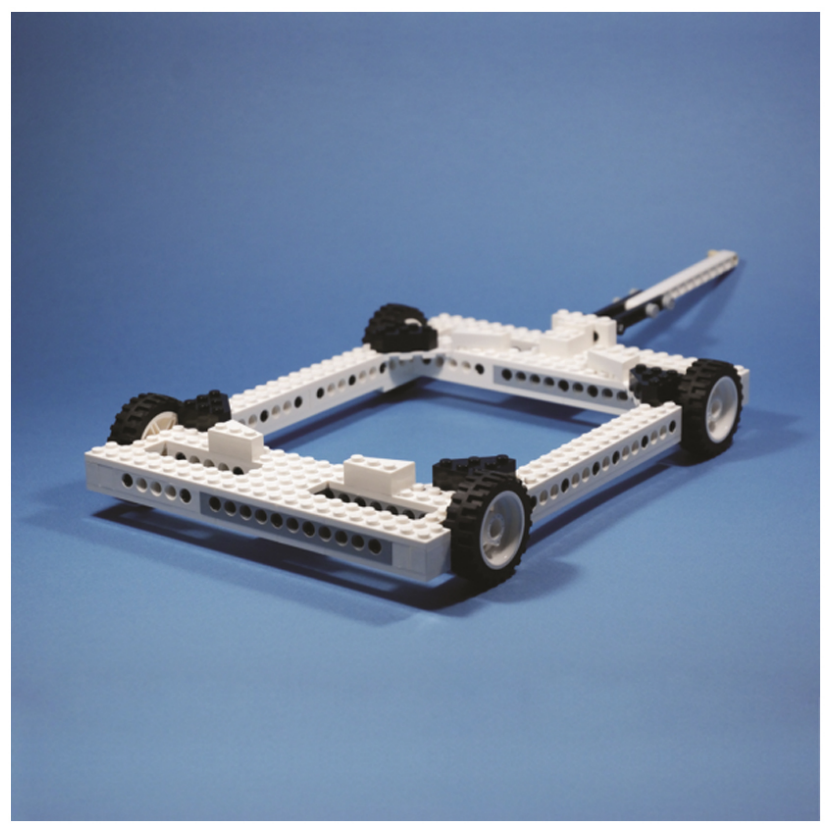

Figure 1. The LEGO cart (The idea of the LEGO cart originated at the department of prof. Hilde Bosmans, KU Leuven, Belgium.) 


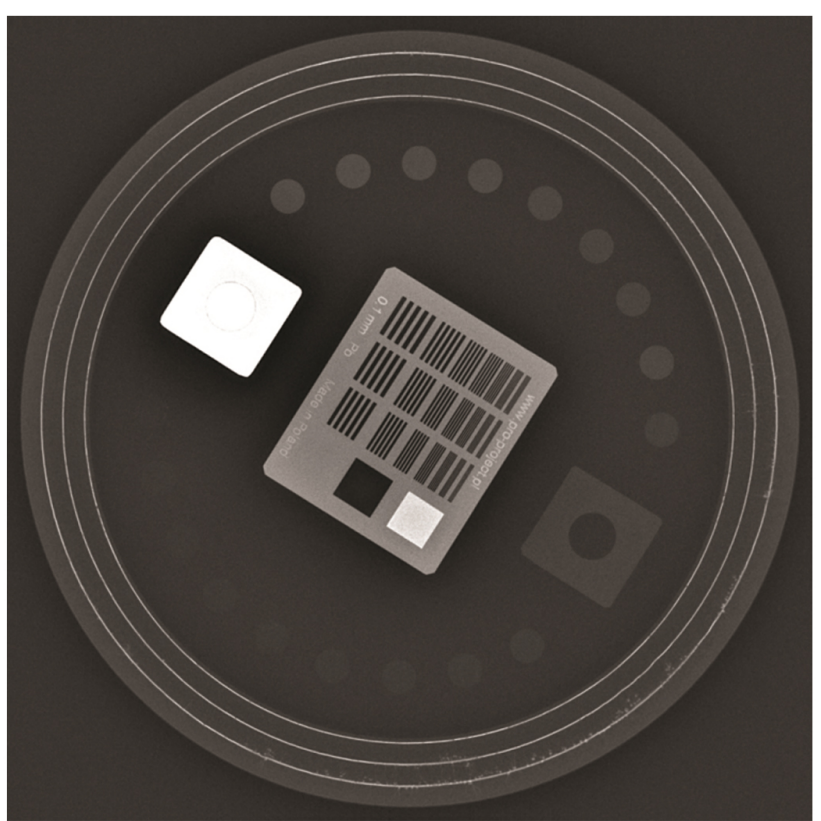

Figure 2. An X-ray image of the phantom Pro-RTG Fluo18
The assessment of image quality was performed using X-ray images acquired in the geometry illustrated in Figures 3 and 4.

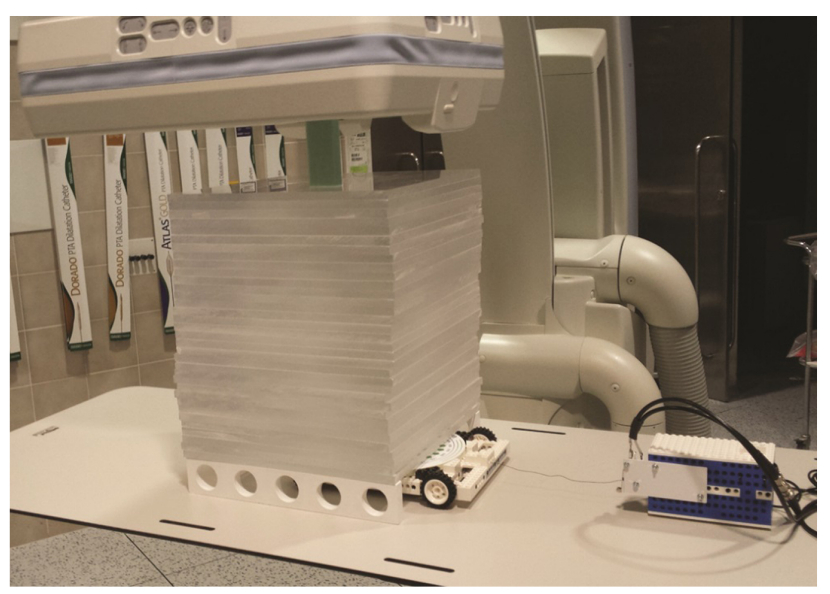

Figure 3. The PMMA, LEGO cart (with its engine) and Pro - RTG Fluo18 in the geometry used

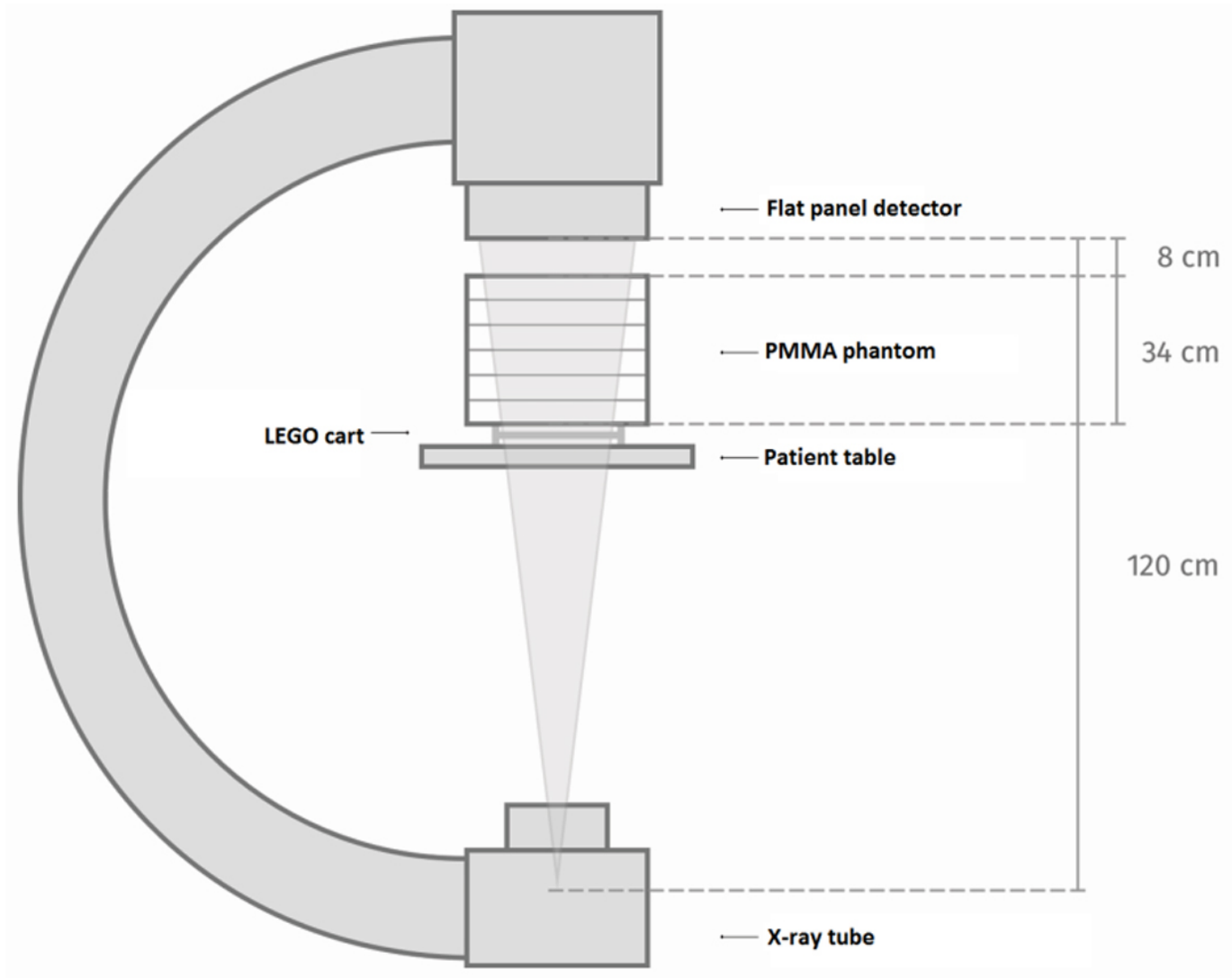

Figure 4. The geometry used by the cine acquisition

The image quality assessment was performed in an abdomi- based indirect conversion flat panel detector of at least $30 \mathrm{~cm}$ nal acquisition mode for following angiography units (each $\times 40 \mathrm{~cm}$, with a pixel size in the range of 154-200 $\mu \mathrm{m}$ and placed in a different hospital), each equipped with a CsI- focus size in the range of 0.6-1.0 mm: GE-Innova IGS 540 
(installed in 2013), Philips - Allura Clarity Xper FD20/20 (installed in 2012, equipped with the ClarityIQ technology), Siemens-Artis Q (installed in 2016), Toshiba-Infinix-i 8000 (installed in 2015). Hereafter, the results are anonymized, so the systems are labelled A, B, C and D. The order of letters does not correspond to the order previously stated, e.g. A is not GE and so on.

All acquisitions were performed in the PA projection for both the dynamic and the static phantom. During the cine acquisition, frame rates of $3 \mathrm{fr} / \mathrm{s}$ or $4 \mathrm{fr} / \mathrm{s}$ (where $3 \mathrm{fr} / \mathrm{s}$ was not possible) were used.

The exposure parameters for each acquisition were recorded together with the air kerma rate. The air kerma rate was calculated from the air kerma rate in the interventional reference point (IRP) for the distance of $70 \mathrm{~cm}$ from the source. The IRP is a fictitious point located $15 \mathrm{~cm}$ from the isocentre towards the source. ${ }^{[19]}$ The air kerma in IRP was taken from the radiation dose structured report, where it is stated for each acquisition.

Image data from each angiography system obtained in DICOM form were sent to our local archiving system. The assessment of the image quality from the point of view of the low-contrast resolution, line-pair resolution and noise was first performed subjectively by two readers, one radiologist and one radiographer, both with more than 10 years of experience. The acquisition images were assessed on a primary medical display, which is used for interpretation of medical images by radiologists. Each reader ordered images of the dynamic phantom from systems A, B, C, and D according to his noise perception and then he assessed low-contrast objects and line-pairs. The subjective assessment was performed during the playback of images for both the static and dynamic phantom. The playback frame rate was the same as the acquisition frame rate, e.g. $3 \mathrm{fr} / \mathrm{s}$ or $4 \mathrm{fr} / \mathrm{s}$ (= normal frame rate). On the static phantom, another test with higher playback frame rate was performed. The playback frame rate was $15-16 \mathrm{fr} / \mathrm{s}$ (= higher frame rate). At this frame rate, eye-brain integration decreases perceived visual image noise and jerkiness, so visibility of low-contrast objects and details is improved..$^{[20,21]}$

Secondly, the objective quantification was performed using the signal difference to noise ratio (SDNR) of low-contrast objects. This quantity has been shown to be useful for optimization of image quality for cardiac imaging. ${ }^{[22]}$ SDNR, as used for image quality analysis, ${ }^{[23-25]}$ was determined for the first nine low-contrast objects (illustrated in Figure 5 in the left part of the image for the second low-contrast object)

Published by Sciedu Press for ten consecutive acquisition images in ImageJ using the following formula (1):

$$
\text { SDNR }=\frac{\mid \text { Signal }_{\text {object }}-\text { Signal }_{\text {background }} \mid}{\sqrt{\left(\sigma_{\text {object }^{2}}{ }^{2}+\sigma_{\text {background }}{ }^{2}\right.}}
$$

where Signal $_{\text {object }}$ was taken as a mean value of the signal of the low-contrast object of the phantom Pro-RTG Fluo18, Signal $_{\text {background }}$ was taken as a mean value of the background signal for an area close to each low-contrast object, $\sigma_{\text {object }}$ is a standard deviation of the low-contrast object signal, and $\sigma_{\text {background }}$ is a standard deviation of the background signal.

SDNR is an important parameter, but it should not be considered separately from the air kerma rate. ${ }^{[4]}$ For comparing images acquired with different air kerma rate, the quantity SDNRD was taken as a useful measure of image quality ${ }^{[23,26]}$ (2):

$$
\text { SDNRD }=\frac{\text { SDNR }}{\sqrt{\text { Air kerma rate }}}
$$

The quantity SDNR has been usually used to describe signal difference or signal contrast, but it does not include any information about the dependence of contrast resolution on a spatial frequency. In this study, the dependence of the contrast resolution on the spatial frequency was determined as a "transmitted contrast", in a similar way as in the study. ${ }^{[27]}$ For each line-pair group, 30 profiles (from six consecutive acquisition images) perpendicular to the line-pair were taken plot, as shown in Figure 5 (with the shorter red line). For each curve, local minimum and maximum values were distinguishable only for some line-pair groups, as shown in Figure 6. In these cases, the "transmitted contrast" was calculated using the following formula:

$$
\text { transmitted contrast }=\frac{\max _{\mathrm{n}-\mathrm{th}}-\min _{\mathrm{n}-\mathrm{th}}}{\max _{1 \mathrm{st}}-\min _{1 \mathrm{st}}}
$$

where $\max _{n-t h}$ is the average of maximal values (five values in each line-pair) of the $n$-th line-pair, $\min _{n-t h}$ is the average of minimal values (four values in each line-pair) of $n$-th line-pair, $\max _{1 \mathrm{st}}$ is the average of maximal values of the first line-pair, $\min _{1 \mathrm{st}}$ is the average of minimal values of the first line-pair. A concept of the transmitted contrast is similar to the concept of the modulation transfer function defined as the dependence of the ratio of image contrast and object contrast on the spatial frequency. The values of transmitted contrast were not normalized to the dose, because according to a study, ${ }^{[28]}$ the increasing dose applied to the detector will not improve the line-pair resolution, so different detector doses should not influence the line-pair resolution. 


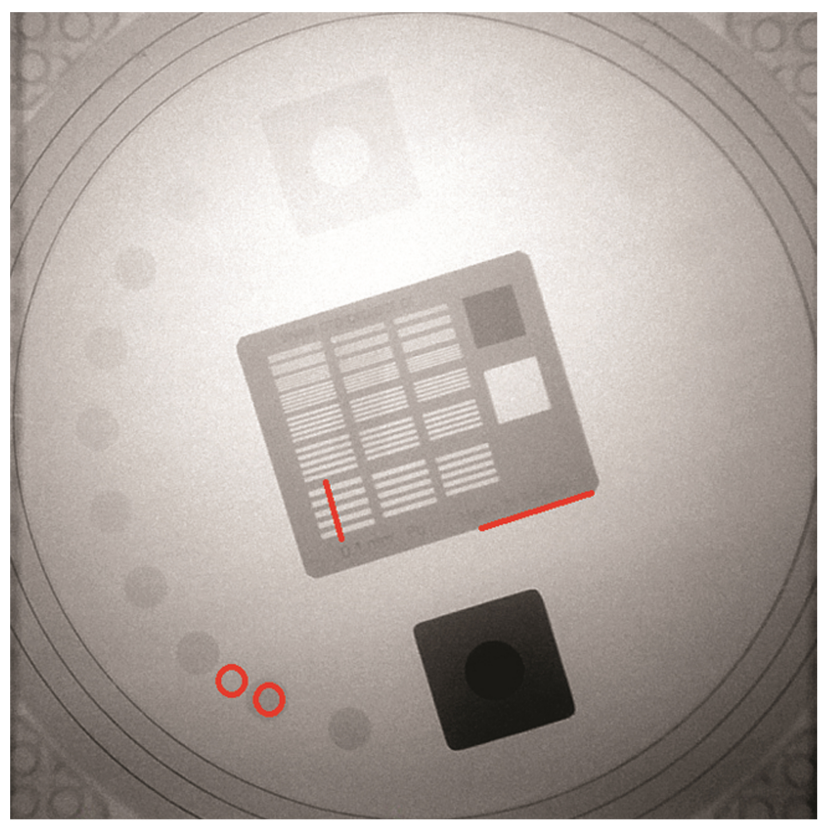

Figure 5. Areas used for the evaluation of low-contrast and line-pair resolutions (red circles - low contrast resolution mark and its background; shorter red line - line-pair resolution (the response illustrated in the following Figure 6; longer red line - the edge)

\section{RESUltS}

The exposure values and air kerma rates are summarized in Table 1.

Focus sizes of the angiography systems used were in the range of 0.6-1.0 mm, but they are not included in Table 1, because the systems would be identifiable, similarly with detector pixel sizes.

The matrix sizes of acquisition images were in the range of $2.5 \mathrm{k} \times 2.0 \mathrm{k}$ to $2.0 \mathrm{k} \times 1.5 \mathrm{k}$, except for System D, where it was only $1.0 \mathrm{k} \times 1.0 \mathrm{k}$ due to a pixel binning. The bit depths of the acquired images were in the range of 14-16 bits. The matrix size of stored images was $1.0 \mathrm{k} \times 1.0 \mathrm{k}$ for all the systems, the bit depths were 10 for System A, 12 for System B, 8 for System C and 12 for System D. Image assessment was based on the stored images. Effect of these differences on the image quality is discussed later.

The images of the dynamic phantom are shown in Figure 7, Figure 8 shows images of the static phantom.

Table 2 summarizes the subjective assessment of the image quality (noise, low-contrast and line-pair resolution) for the dynamic and static phantom.

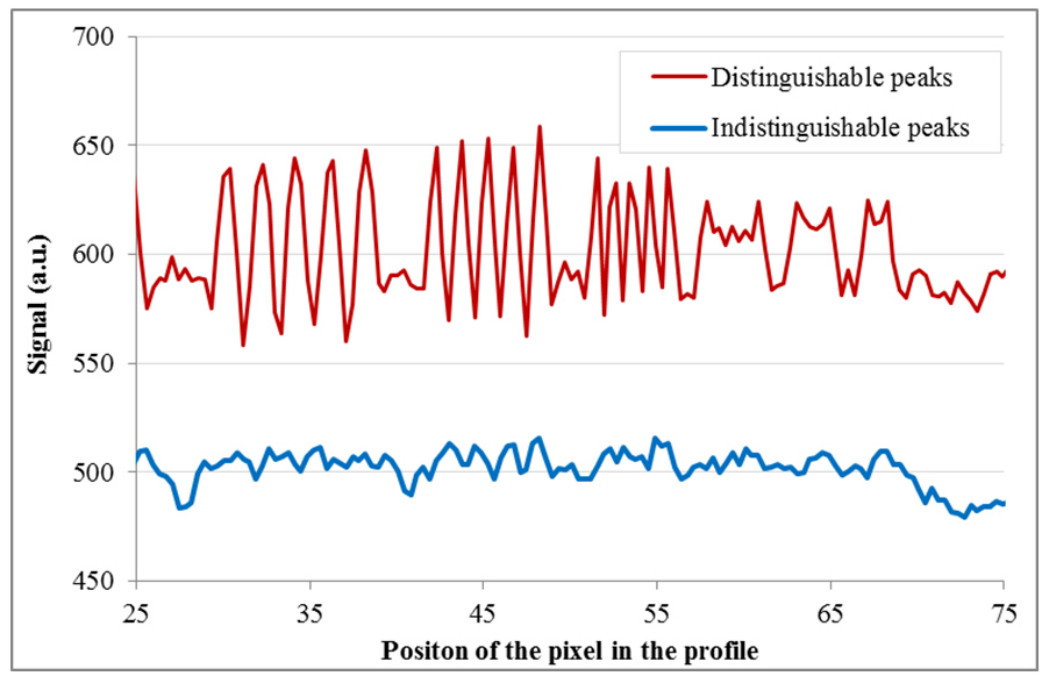

Figure 6. The profile over a few line pairs with local maximum and minimum values (the maximum and minimum values are visible for the first five line pair groups of the red curve, but are not visible for the blue curve)

Table 1. Exposure parameters of cine acquisitions and air kerma rate at the distance $70 \mathrm{~cm}$ from the source

\begin{tabular}{lllll}
\hline Exposure parameters & System A & System B & System C & System D \\
\hline Tube voltage $[\mathrm{kVp}]$ & 103 & 102 & 116 & 98 \\
Tube current*pulse length [mAs] & 61 & $45(615 \mathrm{~mA} * 73 \mathrm{~ms})$ & $7(745 \mathrm{~mA} * 9 \mathrm{~ms})$ & $30(280 \mathrm{~mA} * 106 \mathrm{~ms})$ \\
Spectral filtration & $0.1 \mathrm{~mm} \mathrm{Cu}+1.0 \mathrm{~mm} \mathrm{Al}$ & $0.0 \mathrm{~mm} \mathrm{Cu}$ & -- & $0.1 \mathrm{~mm} \mathrm{Cu}$ \\
Air kerma rate [mGy/s] & System A & System B & System C & System D \\
& 30.9 & 32.7 & 2.3 & 15.4 \\
\hline
\end{tabular}


Table 2. The subjective assessment of the image quality (number of recognizable objects \pm SD)

\begin{tabular}{|c|c|c|c|c|}
\hline & System A & System B & System C & System D \\
\hline Noise & High & Lowest & Highest & Low \\
\hline \multicolumn{5}{|c|}{ Dynamic phantom (Normal frame rate 3-4 fr/s) } \\
\hline No. of low-contrast objects & $10.3 \pm 0.5$ & $12.8 \pm 0.5$ & $9.3 \pm 0.5$ & $10.5 \pm 0.6$ \\
\hline No. of line-pairs & $7.3 \pm 0.5$ & $10.5 \pm 0.6$ & $9.3 \pm 0.5$ & $8.8 \pm 0.5$ \\
\hline \multicolumn{5}{|c|}{ Static phantom (Normal frame rate 3-4 fr/s) } \\
\hline No. of low-contrast objects & $10.5 \pm 0.6$ & $12.8 \pm 0.5$ & $10.3 \pm 0.5$ & $10.8 \pm 0.5$ \\
\hline No. of line-pairs & $12.8 \pm 0.5$ & $12.5 \pm 0.6$ & $10.3 \pm 0.5$ & $11.5 \pm 0.6$ \\
\hline \multicolumn{5}{|c|}{ Static phantom (High frame rate $15-16 \mathrm{fr} / \mathrm{s}$ ) } \\
\hline No. of low-contrast objects & $13.0 \pm 0.0$ & $14.3 \pm 0.5$ & $11.8 \pm 0.5$ & $12.3 \pm 0.5$ \\
\hline No. of line-pairs & $13.0 \pm 0.5$ & $12.8 \pm 0.5$ & $10.3 \pm 0.5$ & $12.3 \pm 0.5$ \\
\hline
\end{tabular}
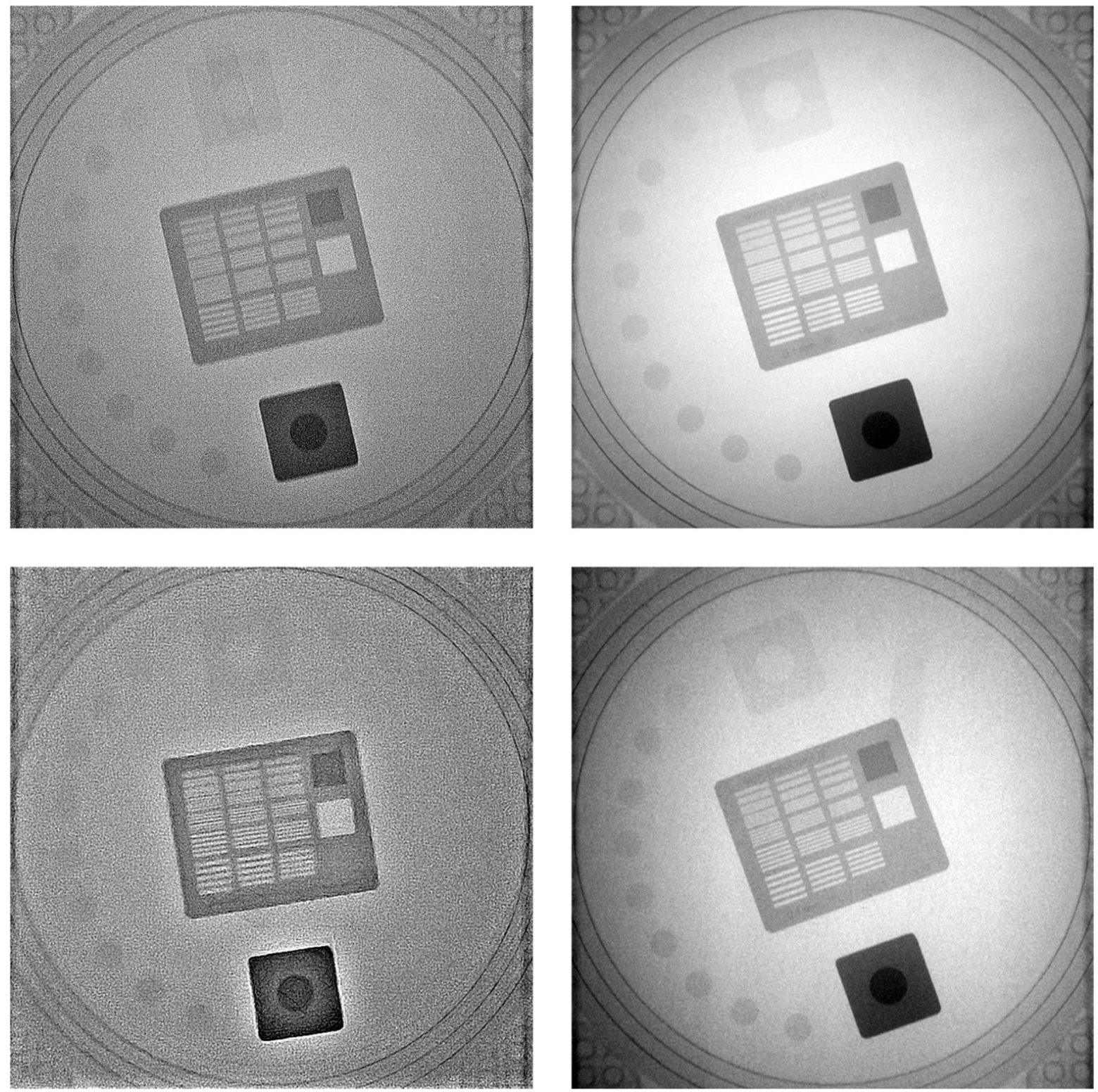

Figure 7. Cine acquisition images of the dynamic phantom - System A (upper left), System B (upper right), System C (bottom left), and System D (bottom right) 

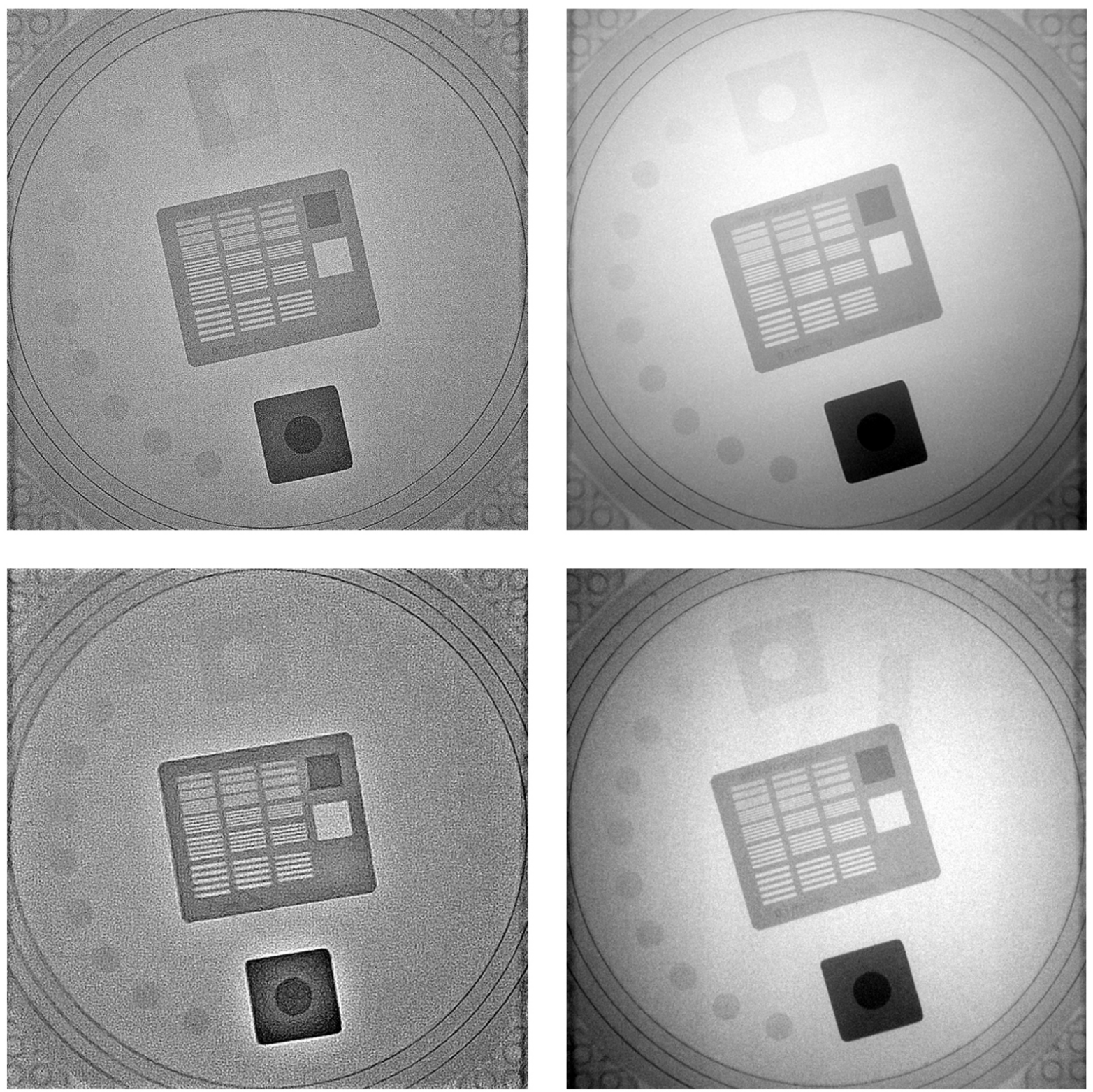

Figure 8. Cine acquisition images of the static phantom - System A (upper left), System B (upper right), System C (bottom left), and System D (bottom right)

The second row of Table 2 includes a visual assessment of noise in the images for each system. An objective value of noise was estimated by taking the standard deviation (SD) of the homogenous area (in the middle area of the image) relative to the mean value of the signal. It could not be taken as the SD only due to the different bit depth of the systems. The SD/mean for Systems A, B, C and D respectively were following: $5.0 \%, 1.7 \%, 4.8 \%$, and $2.1 \%$. An order of systems for objective and subjective measures of noise corresponds with exception of systems A and C, but their SD/mean were very close (5.0\% vs. $4.8 \%)$.

Table 2 includes the number of low-contrast objects and line- pairs for the static and dynamic phantom. Moreover, the number of recognized low-contrast objects and line-pairs are included for the assessment performed at two playback frame rates - 3-4 fr/s and 15-16 fr/s, but only for the static phantom.

Values of SDNR for first nine low-contrast objects of the dynamic and static phantom are shown in Figures 9 and 10, respectively. Values of SDNRD for the first nine low-contrast objects for the dynamic and static phantom are shown in Figures 11 and 12, respectively.

The values of transmitted contrast of the line-pair resolution are shown in Figure 13 for the dynamic phantom and in Fig- 
ure 14 for the static phantom. The last point of each curve corresponds to the last point where the maximum and minimum values were still distinguishable. This value is called a "limiting value".

\section{Discussion}

The exposure values and air kerma rates were summarized in Table 1. System D uses longer frame length and lower tube current for the production of X-ray photons. This approach does not require very high tube loading. The situation is opposite for System C, where the frame length is minimized (as if for cardiac procedures, but neither the acquisition mode nor the angiography system are dedicated to cardiology) and the current is increased. This approach is expected to have a positive effect on the image quality of the dynamic phantom as it should reduce motion unsharpness. However, because of the very low value of the air kerma rate $(2.3 \mathrm{mGy} / \mathrm{s}$ vs. values of $15.4 \mathrm{mGy} / \mathrm{s}$ and higher), a higher noise in the images was expected. The frame length of System A could not be evaluated because the value was not stated in the DICOM file.

The air kerma rate was in the range of 2.3-32.7 mGy/s. As already said, the air kerma rate for System $C$ is very low, but the others correspond to values published in the literature ${ }^{[7]}$ for $36 \mathrm{~cm}$ of PMMA for abdominal acquisition modes of five different angiography systems from three major manufacturers $(17-50 \mathrm{mGy} / \mathrm{s}$ for $4 \mathrm{fr} / \mathrm{s}$, which corresponds to values $13-38 \mathrm{mGy} / \mathrm{s}$ for $3 \mathrm{fr} / \mathrm{s})$.

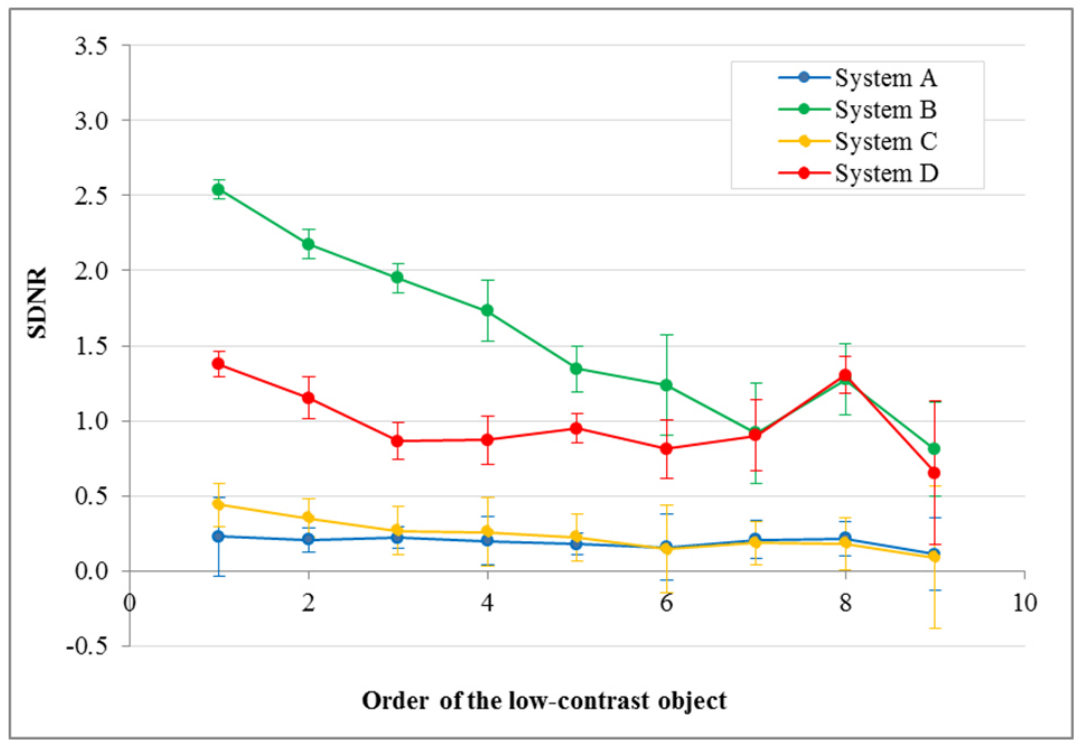

Figure 9. SDNR with relative SD for the cine acquisition of the dynamic phantom

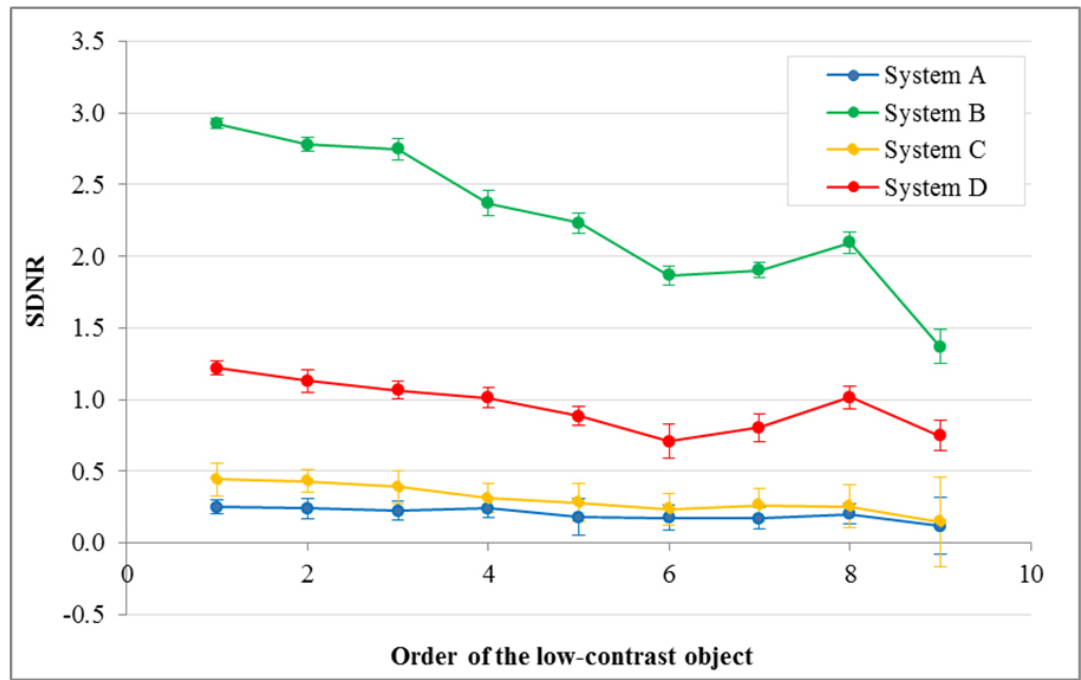

Figure 10. SDNR with relative SD for the cine acquisition of the static phantom 


\subsection{Subjective assessment}

Visual assessment (see Figures 7 and 8) suggests that systems use different postprocessing. Images from Systems $\mathrm{A}$ and $\mathrm{C}$ are similar, as are images from Systems B and D. The images from Systems A and $\mathrm{C}$ are more homogenous from the periphery to the centre, but they suffer from higher noise. The images from Systems B and D are brighter at the centre and darker at the periphery. This might be caused by weaker postprocessing compensation of the scatter radiation. In the image of System $\mathrm{C}$, there appears to be a corona around the objects, indicating that edge enhancement is more pronounced.

Table 2 shows results of the subjective image quality assessment of images for the static and dynamic phantom (unfortunately, \#8 low-contrast object has defective contrast which was verified by another measurement). More low-contrast objects and line-pairs were recognizable on the static phantom. Increasing playback frame rate $15-16 \mathrm{fr} / \mathrm{s}$ improved low-contrast objects recognition by approximately $12 \%-24 \%$, demonstrating eye-brain integration effect on noise.

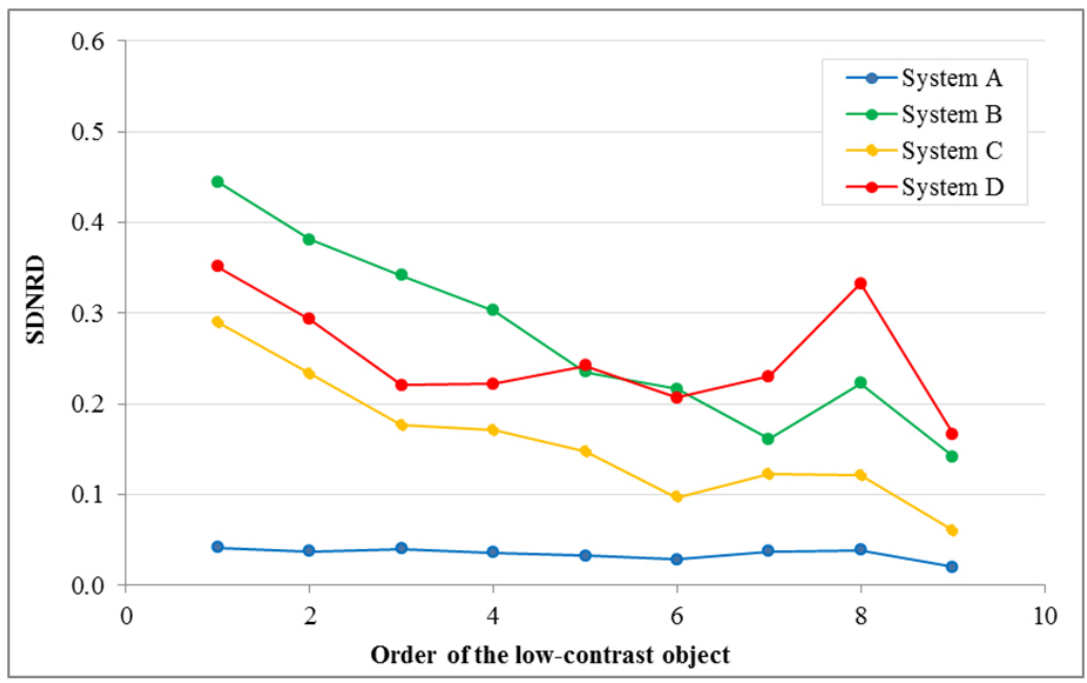

Figure 11. SDNRD for the cine acquisition of the dynamic phantom

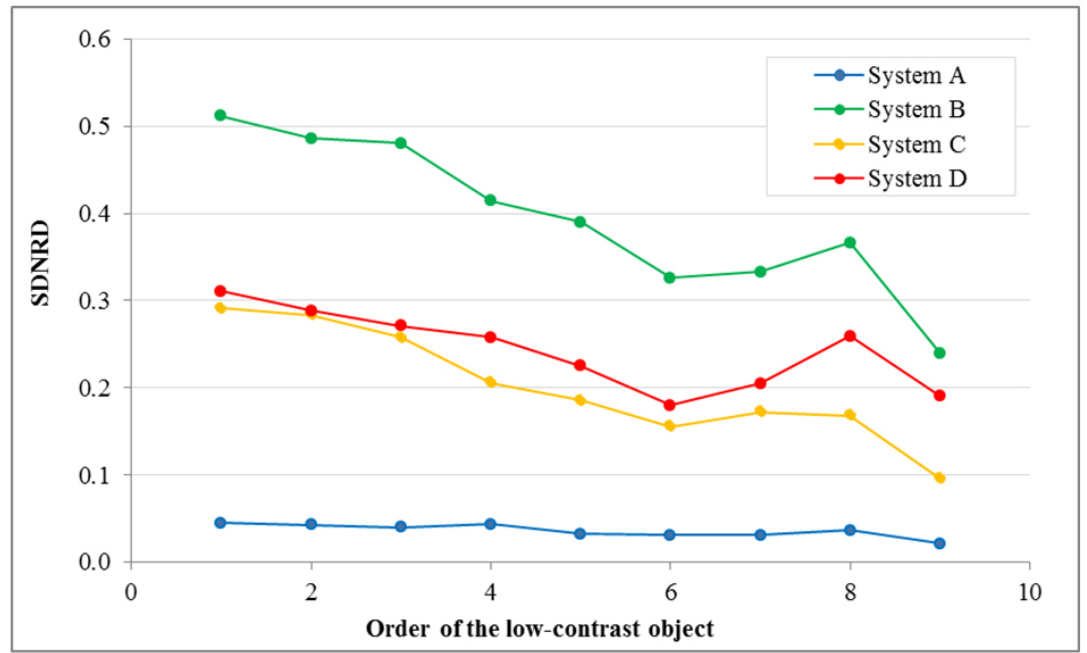

Figure 12. SDNRD for the cine acquisition of the static phantom

\subsection{Objective assessment}

\subsubsection{Noise}

Qualitative and quantitative assessments of noise produced similar results. The least noisy image was from System B
(SD/mean 1.7\%), noisier from System D (SD/mean 2.1\%). The noisiest image was produced by System A (SD/mean $5.0 \%)$; and a similar value was recorded for System C $(\mathrm{SD} / \mathrm{mean} 4.8 \%)$. 


\subsubsection{Signal-difference-to-noise ratio and its values nor-} malized to the dose

As regards the objective assessment, differences in image size need to be discussed. The matrix sizes of stored and assessed acquisition images were different, from $1.0 \mathrm{k} \times 1.0$ $\mathrm{k}$ to $2.5 \mathrm{k} \times 2.0 \mathrm{k}$, bit depths of 8-12 bits. It has been shown that there was no significant contrast variation in the image when the bit depth was reduced from 10 to 8 bits. ${ }^{[29]}$ In another study, albeit primarily on image intensifier technology, authors found an improvement of $20 \%$ in signal-to-noise ratio when the bit depth was lowered from 12 to 8 bits. ${ }^{[24]}$ So we need to take into consideration that lower bit depth can improve the contrast of the image. This could be the case of the System A and C (both 8 bits), but images of both systems proved lower contrast than images from System B and D (both 12 bits). So the different bit depths were not the cause of the worse SDNR.

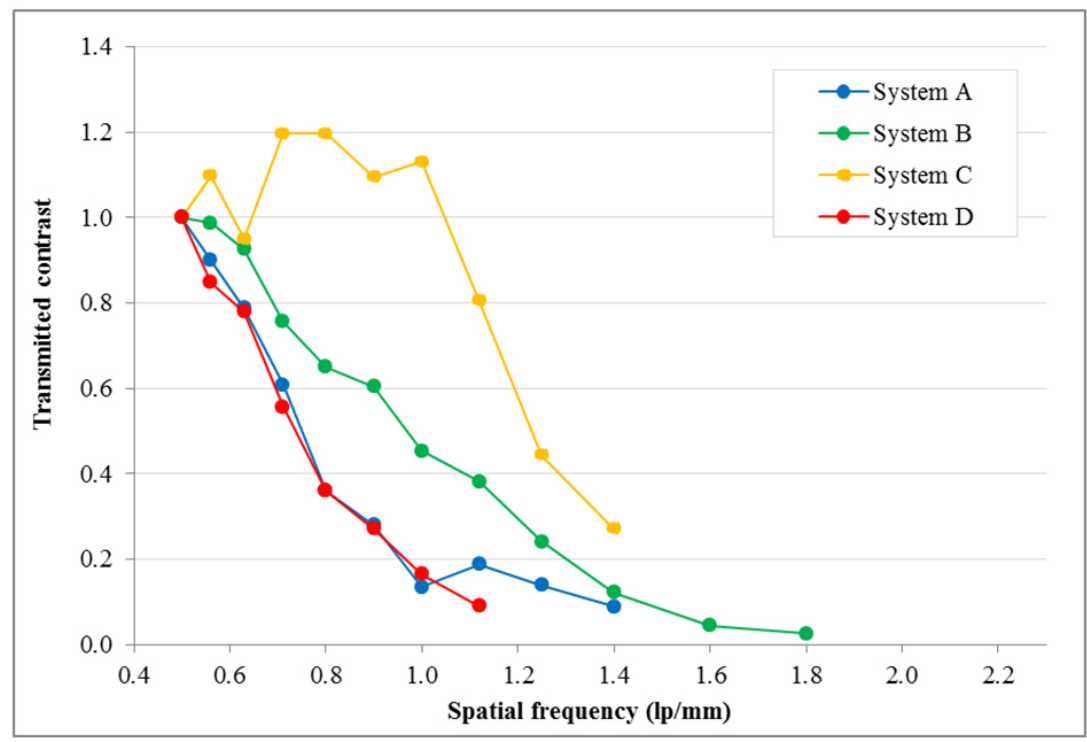

Figure 13. The transmitted contrast of the line pairs of the dynamic phantom

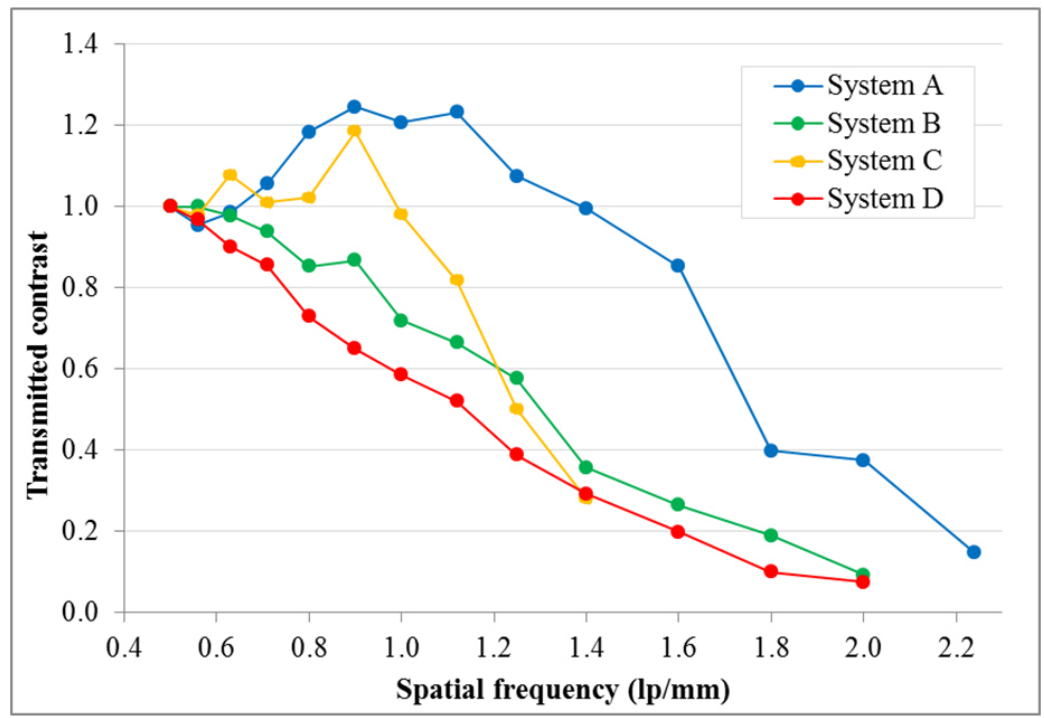

Figure 14. The transmitted contrast of the line pairs of the static phantom

Our assessed image data were all of the size $1.0 \mathrm{k} \times 1.0 \mathrm{k}$, and 10, the best SDNR was produced by System B, the worst so we do not expect any significant effect of the matrix size on the line-pair resolution. As one may see from Figures 9 by Systems A and C. For System C, the low number of recognizable low-contrast objects was caused by the low value 
of the air kerma rate, therefore too high noise was present in the image, but this was not the case of System A, where the air kerma rate was comparable to System B. The order of the systems for the low-contrast resolution (shown in Figures 9 and 10) were similar to the order of images as regards noise. The acquisition images of the static phantom were of similar quality as for the dynamic phantom, with an exception of System B, which provides images of higher SDNR when the static phantom was used.

When SDNRD values (see Figures 11 and 12) were compared (SDNR normalized to the air kerma rate), the image quality was still worst for System A. The contrast of each of the nine low-contrast objects was almost the same and very low. SDNRD for System A reached values only between 0.02 and 0.04 both for the dynamic and static phantom, whilst for System B, SDNRD values were between 0.14 and 0.44 for the dynamic phantom and between 0.24 and 0.51 for the static phantom. At this point, it is not clear why systems A and $C$ give similar SDNR values for objects with different inherent contrast. For Systems A and C, the low-contrast resolution was really poor, roughly 6-11 x worse than for System B.

SDNR was higher for the static phantom than for the dynamic phantom for System B by 15\%-107\%, improvement of SDNR was demonstrated more for the low-contrast objects with lower contrast (objects 6, 7, 8 and 9). For System C, the low-contrast resolution of the static phantom was improved by up to $61 \%$. Similarly as for System B, improvement was better for low-contrast objects with lower contrast. The improvement of the low-contrast objects for the static phantom may be caused by frame averaging.

\subsubsection{Transmitted contrast (spatial resolution)}

For the line-pair resolution, expressed as the transmitted contrast, the results were different for the static and dynamic phantom. As expected, better results were achieved when the phantom was static. The best result for the dynamic phantom (see Figure 13) was achieved by System B (the limiting value $1.80 \mathrm{lp} / \mathrm{mm}$ ); followed by Systems A and C (the limiting value $1.40 \mathrm{lp} / \mathrm{mm}$ for both) and the worst performance was seen on System D (the limiting value $1.12 \mathrm{lp} / \mathrm{mm}$ ). For the static phantom (see Figure 14), the best result was reached by System A (the limiting value $2.24 \mathrm{lp} / \mathrm{mm}$ ), followed by Systems B and D (the limiting value $2.0 \mathrm{lp} / \mathrm{mm}$ for both) and the worst was System C (the limiting value $1.40 \mathrm{lp} / \mathrm{mm}$ ). The limiting values of 1.40 to $1.8 \mathrm{lp} / \mathrm{mm}$ correspond to the limiting spatial resolution value of $1.60 \mathrm{lp} / \mathrm{mm}$ published in the literature ${ }^{[4]}$ for the PMMA of the similar water equivalent thickness.

Although the frame length was shortest for System C, the limiting value of line-pair resolution for the dynamic phantom was worse compared to System B. This may be affected by noise (but only up to a certain point) which was very high. Even though the spatial resolution is usually not usually affected by a detector dose, ${ }^{[28]}$ at some point high noise will have an impact and this may be occurring here under conditions simulating obese patients. Other factors causing the lower line-pair resolution of System $\mathrm{C}$ may be higher energy, therefore more scatter, and larger focus size used than for System B. For System D, the longest frame length was used, but the line-pair resolution of the static phantom did not suffer from it significantly. Effect of the long frame length was evident for the dynamic phantom for System D, as well as for System A.

Results were not corrected for the focal sizes because the longer frame length was compensated by the smaller focus size (the frame length was inversely proportional to the focus size for systems B, C, D), so each system tried to reduce the unsharpness.

Differences between System A for the static and dynamic phantom were probably caused by the long frame length which led to the motion unsharpness. This unsharpness did not occur for the static phantom, where the line-pair resolution was much better. The longer frame length may be caused by the X-ray tube performance limitation, as the tubes did not have the same nominal anode power values.

The shape of the curve (decreasing, then increasing) for the system C in Figures 13 and 14 (both the dynamic and static phantom) was unexpected. This is most likely a result of postprocessing, mainly high pass filtering. ${ }^{[7,30,31]}$ This effect appeared also for System A for the static phantom.

\subsubsection{The edge response}

In order to gain greater understanding of how the postprocessing influences the image, the edge response was calculated. The edge response was calculated as an average value of 15 profiles drawn perpendicularly to the edge (the edge is highlighted by the longer red line in Figure 5). The edge responses are shown in Figure 15 for all systems. The values of the signal in each position on the profile were normalized to the average value of a homogeneous area before the edge. In an ideal case, the values on the profile should be equal to 1.0 before the profile (due to the normalization) and then there should be a steep increase to another value (which will be different for each system). The value behind the edge should be ideally the same as in the edge.

It is evident from Figure 15 that the images from Systems $A$ and $C$ suffer from higher noise, as was seen in the image quality assessments earlier. For the curves from Systems A 
and $\mathrm{C}$, there is an obvious steep decrease in front of the edge and a steep decrease behind the edge, which are caused by the postprocessing (edge enhancement). On the other hand, Systems B and D do not use as strong postprocessing, and their average values before the profile and after the profile are more constant.

\subsubsection{Interpretation of results}

Care must be taken in interpreting the results as an absolute indication of image quality performance in clinical practice. Image processing is not designed to operate on these test objects and we cannot assume they are displayed to their best advantage. However, the results do add important information which allows us gain a fuller assessment of image quality. When talking to clinicians performing abdominal procedures, all were pleased with the image quality. Clinicians considered all systems to provide sufficient diagnostic image quality and could not readily offer detailed information on how each system works, or how their performance varies with patient size. Imaging test objects in clinical modes can give additional data on these points. In addition, once reproducible data is obtained, it can also be used for performance trending and for assessing if performance is maintained after repair actions.

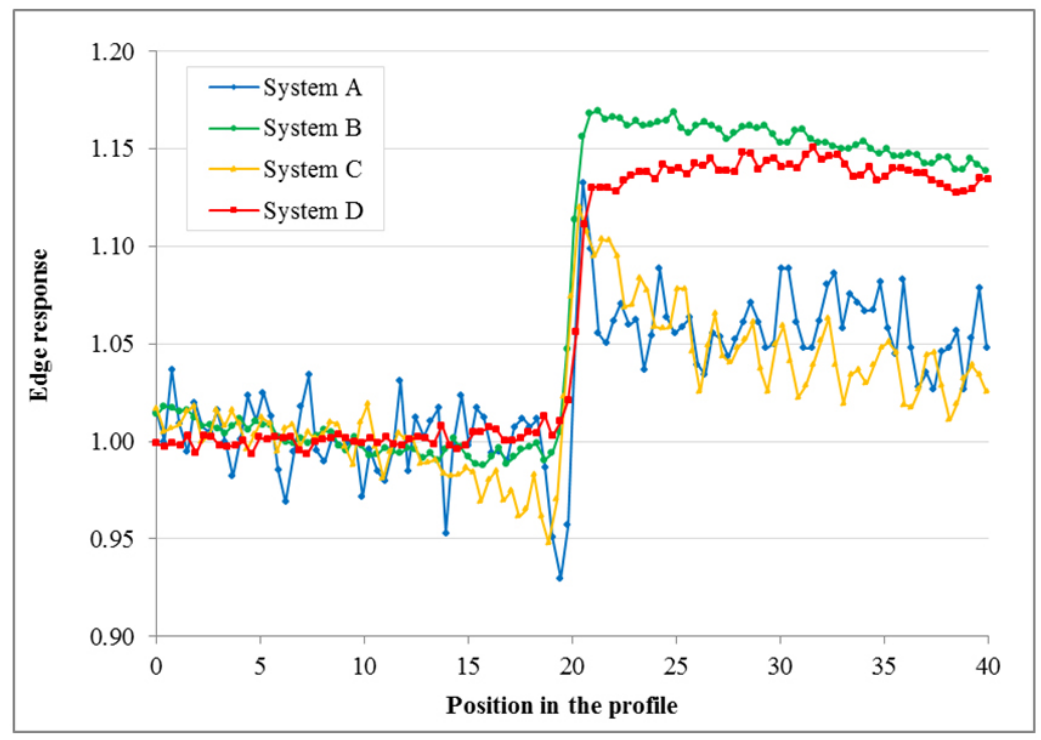

Figure 15. The profile of the edge response

Abdominal procedures are performed on System C less frequently, so this system may not be optimized for obese patients, as indicated by the low air kerma rate and poor image quality in our study.

Image quality assessment showed performance differences across the acquisition modes of the four tested angiography systems. There were differences between the line-pair resolution for the dynamic and static phantom for each of the systems, but the greatest differences were found for System A. The results indicate that tube performance limitations may be a limiting factor for some systems as attenuation is increased. The best system as regards low-contrast resolution and noise was System B, followed by System D. The images from System A and C showed reduced SDNR and increased noise, which negatively influences visibility of the low-contrast objects. Higher noise might be caused by the quite low air kerma rate for System C (see Table 1), but there is no explanation for the poor image quality of System A. Processing may play a part in these responses and care needs to be taken in inferring clinical performance from results in non-anthropomorphic phantoms. However, the results point to potential for imaging optimization in some of the systems.

\section{ACKNOWLEDgeMENTS}

The authors would like to thank the staff of the Dept. of Radiologic Methods and Nuclear Medicine, Faculty Hospital Brno; Dept. of Diagnostic Radiology, University Hospital Hradec Kralove; Dept. of Radiology, University Hospital Olomouc; Dept. of Radiology, Hospital Usti nad Orlici for support during measurements. The authors are deeply indebted to Colin Walsh for helpful discussions.

\section{CONFLiCTS OF INTEREST Disclosure}

The authors declare that they have no competing interests. 


\section{REFERENCES}

[1] Hammond KL. Practical issues in the surgical care of the obese patient. Ochsner J. 2013; 13(2): 224-227.

[2] Bushberg JT, Seibert JA, Leidholdt Jr. EM, et al. The Essential Physics of Medical Imaging. Lippincott Williams \& Wilkins, Philadelphia; 2012.

[3] American Association of Physicist in Medicine. Functionality and operation of fluoroscopic automatic brightness control/automatic dose rate control logic in modern cardiovascular and interventional angiography systems; 2012.

[4] Dehairs M, Bosmans H, Desmet W, et al. Evaluation of automatic dose rate control for flat panel imaging using a spatial frequency domain figure of merit. Phys Med Biol. 2017; 62: 6610-6630. PMid:28632501 https ://doi .org/10.1088/1361-6560/aa7a $9 d$

[5] Gislason-Lee A, Hoornaert B, Cowen AR, et al. Understanding automated dose control in dynamic X-ray imaging. European Congress of Radiology. Vienna, Austria. 2013.

[6] Lin PJP. The operation logic of automatic dose control of fluoroscopy system in conjunction with spectral shaping filters. Med Phys. 2007; 34(8): 3169-3172. PMid:17879779 https ://doi.org/10.1118/ 1.2752576

[7] Wunderle KA, Rakowski JT, Dong FF. Approaches to interventional fluoroscopic curves. Journal of Applied Clinical Medical Physics. 2016; 17(1): 342-352. PMid:26894349 https://doi.org/10.1 120/jacmp.v17i1.5788

[8] Dance DR, Christofides S, Maidment ADA, et al. Diagnostic radiology physics: A handbook for teachers and students. International Atomic Energy Agency. 2014.

[9] Seibert JA. Digital fluoroscopy imaging: Acquisition, processing \& display. AAPM 46th annual meeting; Pittsburgh, PA: 2004.

[10] Toshiba's newest advanced image processing technology brings clinical advantages to interventional imaging. [cited 2017 June 6]. Available from: http://www.toshibamedicalsystems.com/pr oducts/dose/lowdose_xray/index.html

[11] Philips AlluraClarity: Enhancing image quality and reducing dose. [cited 2017 June 6]. Available from: http://www.mdbuyline.com/philips-alluraclarity -enhancing-image-quality-reducing-dose/

[12] CARE+CLEAR. Improving image quality and optimizing dose in every Artis system. [cited 2017 June 10]. Available from: https://www.healthcare.siemens.cz/angio/innovation s-technologies/care-clear

[13] Tapiovaara M. Objective measurement of image quality in fluoroscopic X-ray equipment: FluoroQuality. 2003.

[14] Guzzaloni G, Minocci A, Marzullo P, et al. Sagittal abdominal diameter is more predictive of cardiovascular risk than abdominal fat compartments in severe obesity. Int J Obes. 2009; 33(2): 233-238. PMid:19139755 https://doi.org/10.1038/ijo.2008.271

[15] Sugerman HJ. Pathophysiology of obesity comorbidity: The effects of chronically increased intraabdominal pressure. In: Schauer PR, Schirmer BD, Brethaues SA (eds). Minimally invasive bariatric surgery; Springer; 2007.

[16] Lambert DM, Marceau S, Forse A. Intra-abdominal pressure in the morbidly obese. Obesity Surgery. 2005; 15: 1225-1232. https: //doi.org/10.1381/096089205774512546
[17] X-ray mass attenuation coefficients. NIST Physical Measurement Laboratory. [cited 2017 May 25]. Avalilable from: http://www.ni st.gov/pml/data/xraycoef/index.cfm

[18] Suramo I, Paivansalo M, Myllyla V. Cranio-caudal movements of the liver, pancreas and kidneys in respiration. Acta Radiol Diagn. 1984; 25(2): 129-131. https://doi.org/10.1177/02841851840250 0208

[19] International Electrotechnical Commission. Medical electrical equipment - Part 2-43: Particular requirements for the basic safety and essential performance of X-ray equipment for interventional procedures. IEC. 2010.

[20] Hirshfeld JW Jr, Balter S, Brinker JA, et al. ACCF/AHA/HRS/SCAI clinical competence statement on physician knowledge to optimize patient safety and image quality in fluoroscopically guided invasive cardiovascular procedures: A report of the American College of Cardiology Foundation/American Heart Association/American College of Physicians Task Force on Clinical Competence and Training. Circulation. 2005; 111(4): 511-532. PMid:15687141 https: //doi.org/10.1161/01.CIR.0000157946.29224.5D

[21] Allisy-Roberts P, Williams J. Farr's physics for medical imaging. Saunders Ltd; 2008. ISBN 978-0702028441.

[22] Gislason AJ, Davies AG, Cowen AR. Dose optimization in pediatric X-ray imaging. Med Phys. 2010; 37(10): 5258-5269. PMid:21089760 https ://doi.org/10.1118/1.3488911

[23] Tanguay J, Kim HK, Cunningham IA. The case for single-exposure angiography using energy resolving photon-counting detectors: A theoretical comparison of signal and noise with conventional subtraction angiography. Proc of SPIE. 2011.

[24] Vano E, Ubeda C, Geiger B, et al. Influence of image metrics when assessing image quality from a test object in cardiac X-ray systems. J Digit Imaging. 2011; 24(2): 331-338. PMid:20127268 https ://doi.org/10.1007/s10278-009-9268-7

[25] Hwang YS, Tsay HY, Chen CC, et al. Survey of radiation dose, image quality and equipment performance of mammography units in Taiwan. Dössel O and Schlegel WC (eds.): WC. 2009.

[26] Kachelriess M. Understanding image quality and radiation dose in MDCT and CBCT. European Congress of Radiology. Vienna, Austria. 2015.

[27] Droege RT, Morin RL. A practical method to measure the MTF of CT scanners. Med Phys. 1982; 9(5): 758-760. PMid:7155079 https://doi.org/10.1118/1.595124

[28] Körner M, Weber CH, Wirth S, et al. Advances in digital radiography: Physical principles and system overview. Radiographics. 2007; 27(3): 675-686. PMid:17495286 https ://doi.org/10.1148/rg .273065075

[29] Sanchez R, Vano E, Ubeda C, et al. Influence of image metrics when assessing image quality from a test object in cardiac X-ray systems: Part II. J Digit Imaging. 2012; 25(4): 537-541. PMid:22223157 https ://doi.org/10.1007/s10278-011-9448-0

[30] Lanca L, Silva A. Digital imaging systems for plain radiography. Springer; 2013.

[31] Davidson RA. Radiographic contrast-enhancement masks in digital radiography. Chapter 5: Current post-processing methods in digital radiography [dissertation]: University of Sydney; 2006. 